1997-01-01

\title{
On the dynamic behaviour of a thermoviscoelastic body in frictional contact with a rigid obstacle
}

Kenneth Kuttler

klkuttle@math.byu.edu

K. T. Andrews

M. Shillor

Follow this and additional works at: https://scholarsarchive.byu.edu/facpub

Part of the Mathematics Commons

Original Publication Citation

European Journal of Applied Mathematics, 8(1997), pp 417-436.

\section{BYU ScholarsArchive Citation}

Kuttler, Kenneth; Andrews, K. T.; and Shillor, M., "On the dynamic behaviour of a thermoviscoelastic body in frictional contact with a rigid obstacle" (1997). Faculty Publications. 671.

https://scholarsarchive.byu.edu/facpub/671

This Peer-Reviewed Article is brought to you for free and open access by BYU ScholarsArchive. It has been accepted for inclusion in Faculty Publications by an authorized administrator of BYU ScholarsArchive. For more information, please contact ellen_amatangelo@byu.edu. 


\title{
On the dynamic behaviour of a thermoviscoelastic body in frictional contact with a rigid obstacle
}

\author{
K. T. ANDREWS ${ }^{1}$, K. L. KUTTLER ${ }^{2}$ and M. SHILLOR ${ }^{1}$ \\ ${ }^{1}$ Department of Mathematical Sciences, Oakland University, \\ Rochester, MI 48309-4401, USA \\ ${ }^{2}$ Department of Mathematical Sciences, Michigan Technological University, \\ Houghton, MI 49931, USA
}

(Received in revised form 1 October 1996)

\begin{abstract}
We consider the dynamic behaviour of a thermoviscoelastic body which may come into frictional contact with a rigid obstacle. The frictional contact is modelled by general contact and friction laws which include as special cases the power law normal compliance condition and the corresponding generalization of Coulomb's law of dry friction. The stress-strain constitutive relation is assumed to be of Kelvin-Voigt type and the frictional heat generation on the contact surface is taken into account. In this setting we establish the existence of a solution to a weak version of the energy-elasticity system which consists of a parabolic equation coupled with a variational inequality.
\end{abstract}

\section{Introduction}

Frictional contact between parts and structures that is accompanied by heat generation is a common feature in many technological settings. Such contact occurs, for example, in the operation of brakes, valves, pistons and transmissions. Consequently, the modelling and analysis of the dynamic behaviour of such systems is of considerable applied interest, as a first step toward the goal of achieving optimal design and control. In addition, the noise and vibration characteristics of such systems have been the subject of increased attention. As a result, in recent years there has been a substantial development in the mathematical literature devoted to this topic. General models for the dynamic behaviour of viscoelastic bodies can be found in Germain [1], Duvaut \& Lions [2] and in Maugin [3], among other places. The mathematical analysis of such models is well advanced in cases where the standard boundary conditions are used. However, in cases where contact or impact are involved, the boundary conditions become more complicated and the mathematical analysis more involved. The use of Signorini's contact condition leads, for example, to a system of variational inequalities for which existence results, for one-dimensional dynamic problems, have been obtained only recently by Do [4], Kim [5] and Kuttler [6].

The situation becomes still more complicated when thermal effects are included in the model. Recent results for such one-dimensional problems may be found in References 7 10. However, in all these papers, the contact is assumed to be frictionless and the boundary condition for the temperature on the contacting surface is one of the standard types. The paucity of results even in these simple geometric settings illustrate the mathematical 
difficulties encountered in using unilateral constraints in second-order evolution problems. From the physical point of view unilateral conditions can also be seen as unrealistic, in that they imply that there is no mutual penetration of the contacting bodies and hence they ignore the deformation of asperities that contact causes. Consequently, in recent years investigators have explored alternative approaches to modelling the contact interface. For example, Oden \& Martins [11] have proposed a model where the contact interface has a normal compliance characterized by a power-law relationship between the normal pressure and the penetration. Experimental justification for such a relationship in the case of metallic interfaces subject to low pressures may be found in Reference 12. Some examples of existence and uniqueness results for initial-boundary value problems that incorporate this condition may be found in References 13-15. The most general results in this area may be found in Reference 15, which treats the case of frictional contact, but which does not allow for frictional heat generation on the contacting surface. However, in many applications it is essential for the effects of heat generation to be taken into account. For example, the sudden application of car brakes may lead to the dissipation of energy in the form of frictional heating at a rate of as much as $300 \mathrm{HP}$.

In this paper, we prove the existence of a weak solution to a dynamic $n$-dimensional thermoviscoelastic contact problem that does allow for such heat generation. We assume general contact and friction conditions which include as special cases versions of the power law normal compliance and friction conditions of Oden and Martins. Thus our formulation allows for a coefficient of friction which may depend on the contact stress. Our assumptions on the initial and boundary data are less restrictive than those imposed in Reference 16, but our growth assumptions on the contact and friction conditions are more restrictive. Further results on the regularity of solutions should help with more realistic assumptions. The proof involves the application of an abstract existence theorem for evolution equations to an approximation problem that contains a regularized friction functional. We then obtain an a priori estimate on the solutions to such problems, which permits passage to the limit and the establishment of the existence result. Thus, the regularized problems may be used as the basis for constructing numerical approximations to the original problem.

The fact that this estimate alone implies sufficient regularity to obtain an existence result is due to the presence of viscosity. By contrast, in the absence of viscosity, the process of passing to the limit in dynamic problems that contain Signorini's frictionless contact condition is much more delicate, even in the one-dimensional case, and requires a continuity property of the wave operator that is a consequence of the so-called divcurl lemma of compensated compactness [5, 8, 9]. Indeed, the existence and uniqueness of solutions to the $n$-dimensional dynamic contact problem using Signorini's frictionless condition and no viscosity remains an open problem for $n>1$.

The existence and uniqueness results established in this paper open the way to a more detailed analysis of dynamic frictional contact problems. For example, issues such as the growth and stability of hot spots will require further investigation into the regularity properties of solutions. Efficient numerical algorithms and numerical simulations will be required to make models of this behaviour useful in industrial design processes.

We now describe the remaining sections of this paper. \$2 contains a description of the physical model and the governing energy-elasticity system of equations. $\$ 3$ gives a 


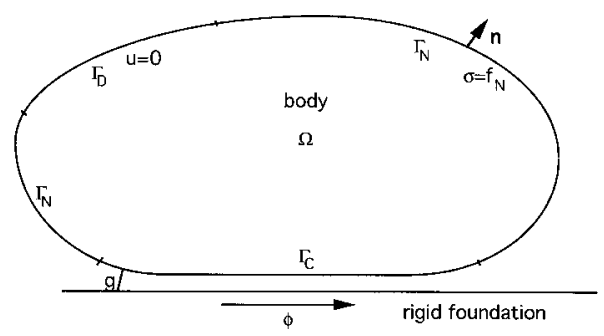

FIGURE 1. The viscoelastic body.

weak formulation of the problem which takes the form of a variational inequality for the displacement coupled with an equation for the temperature. The presence of a variational inequality is to be expected for a problem involving dynamic frictional contact, since friction laws are naturally expressed as inequalities while dynamic contact conditions create discontinuities in the surface velocity and thus necessitate a weak formulation of the problem. This section also contains a statement of the main existence result, Theorem 3.2. In $\S 4$ we describe the approximation problem and obtain its solution by applying an abstract existence theorem. We also obtain a priori an estimate on the solution of such problems. This estimate is used in $\S 5$ to pass to the limit and thus establish the main result. In $\S 6$, we prove a uniqueness result under additional conditions on the data, and in $\S 7$, we give our conclusions.

\section{The model}

In this section we present the physical setting and formulate the model as a coupled system of partial differential equations.

We follow the exposition of the model set forth in [6], and employ the so-called KelvinVoigt viscoelasticity law. We retain the inertial terms in the elasticity equations, and thus consider the full dynamic problem. The behaviour of the bulk material is assumed to be linear, and the only (strongly) nonlinear effects that are considered occur on the part of the boundary which may contact a rigid foundation.

The physical setting consists of a viscoelastic body, represented (in its reference configuration) by $\Omega$, a region in $\mathbb{R}^{m},(m=2,3)$, whose boundary surface $\partial \Omega=\Gamma$ is divided into three disjoint parts. On the part denoted by $\Gamma_{D}$ the body is supposed to be clamped while on the part denoted by $\Gamma_{N}$ known tractions act on the body. Finally, on the part denoted by $\Gamma_{C}$ the body may come into frictional contact with a rigid foundation $\Gamma_{R}$, which is situated $g$ units away, as measured in the direction normal to $\Gamma_{C}$. As usual we call $g$ the gap function. We also allow for the possibility that the rigid foundation moves with a prescribed tangential velocity $\phi$. This setting is depicted in Fig. 1. The reference configuration is assumed to be stress free and at a constant temperature, conveniently set as zero. The temperature below is measured with respect to this reference temperature.

Let $f_{A}=\left(f_{A 1}(x, t), \ldots, f_{A m}(x, t)\right)$ be the (nondimensional) density of the applied body forces acting in $\Omega$, and let $f_{N}=\left(f_{N 1}(x, t), \ldots, f_{N m}(x, t)\right)$ be the tractions that are applied on $\Gamma_{N}$. For simplicity, we assume below that the density of the material is constant, and we set it equal to one. Let $q$ denote the density of the prescribed body heat sources. Then if 
$u=\left(u_{1}(x, t), \ldots, u_{n}(x, t)\right), \theta=\theta(x, t)$, and $\sigma=\left\{\sigma_{i j}(x, t)\right\}$ represent the displacement vector, temperature and stress tensor at location $x$ and time $t$, then the system of viscoelastic and energy equations takes the (nondimensional) form

$$
\begin{aligned}
u_{i, t t}-\sigma_{i j, j} & =f_{A i}, \\
\theta_{, t}-\left(k_{i j} \theta_{, j}\right)_{, i} & =-c_{i j} u_{i, t j}+q .
\end{aligned}
$$

Here $i, j=1, \ldots, m$, and the repeated index convention is employed. The portion of a subscript prior to a comma indicates a component, while the portion after the comma refers to a partial derivative. The constants $c_{i j}$ are the components of the tensor of thermal expansion and the constants $k_{i j}$ are the components of the tensor of thermal conductivity. We take for the stress-strain relation the Kelvin-Voigt thermoviscoelastic law

$$
\sigma_{i j}=a_{i j k l} u_{k, l}+b_{i j k l} u_{k, t l}-c_{i j} \theta .
$$

Here $a=\left\{a_{i j k l}\right\}$ and $b=\left\{b_{i j k l}\right\}$ are the tensors of elastic and of viscoelastic coefficients, respectively.

We now describe the initial and boundary conditions. The initial conditions take the form

$$
u(0, \cdot)=u_{0}, \quad u_{, t}(0, \cdot)=v_{0}, \quad \theta(0, \cdot)=\theta_{0} .
$$

To describe the boundary conditions we introduce the unit normal $\mathbf{n}=\left(n_{1}, \ldots, n_{m}\right)$ to $\partial \Omega$. It is assumed throughout this paper that $\partial \Omega$ is Lipschitz, and therefore $\mathbf{n}$ exists at almost every point of the boundary. We then let

$$
\sigma_{n}=\sigma_{i j} n_{i} n_{j}, \quad u_{n}=u_{i} n_{i}
$$

be the normal components of $\sigma$ and $u$ on $\Gamma$, and let

$$
\sigma_{T}=\sigma \mathbf{n}-\sigma_{n} \mathbf{n}, \quad u_{T}=u-u_{n} \mathbf{n},
$$

be the tangential components. Then on the $\Gamma_{D} \cup \Gamma_{N}$ portion of the boundary we impose the following conditions:

$$
\begin{array}{cl}
u=0 & \text { on } \Gamma_{D}, \\
\sigma \mathbf{n}=f_{N} & \text { on } \Gamma_{N}, \\
\theta=\theta_{b} & \text { on } \Gamma_{D} \cup \Gamma_{N} .
\end{array}
$$

Finally, we consider the boundary conditions on the potential contact surface $\Gamma_{C}$, which is where our main interest lies. Mechanically we assume a general contact law which includes the power law normal compliance condition as a special case, i.e.

$$
-\sigma_{n}=p\left(\cdot, u_{n}-g\right) \quad \text { on } \Gamma_{C},
$$

where $p=p(x, r), x \in \Gamma_{C}, r \in R$ is a given nonnegative function which satisfies additional growth and smoothness conditions listed below. Such a contact law may be viewed as a mathematical regularization of the Signorini contact condition in the following sense. If we take $p(x, r)=\lambda \max \{r, 0\}$ in (2.8) and allow $\lambda \rightarrow \infty$, then formally we recover the Signorini condition

$$
u_{n} \leqslant g, \quad \sigma_{n} \leqslant 0, \quad \sigma_{n}\left(u_{n}-g\right)=0 .
$$


However, as previously indicated, there are also physical considerations involving the deformation of asperities in response to the normal load that suggest that the contact interface has a normal compliance characterized by a power law relation (see elsewhere [11-13, $15,17]$ ) for details). In contrast to Signorini's nonpenetration condition $u_{n} \leqslant g$, a power law relation allows for interpenetration to occur in the presence of high contact stress.

The corresponding generalization of Coulomb's law of dry friction may be stated as

$$
\begin{array}{ll}
\left|\sigma_{T}\right| \leqslant F\left(\cdot, u_{n}-g\right) & \text { on } \Gamma_{C}, \\
\left|\sigma_{T}\right|<F\left(\cdot, u_{n}-g\right) & \text { implies } u_{T, t}=\phi, \\
\left|\sigma_{T}\right|=F\left(\cdot, u_{n}-g\right) & \text { implies } u_{T, t}=\phi-\lambda \sigma_{T} .
\end{array}
$$

Here $F=F(x, r), x \in \Gamma_{C}, r \in R$ is a given function (called the friction bound), $\phi$ is the tangential velocity of the rigid foundation, and $u_{T, t}$ is the tangential velocity of the body. The conditions (2.10)-(2.11) may be interpreted physically in the following way. Condition (2.10) may be said to characterize the behaviour of the body in the so-called stick zone. It implies that when the applied tangential stress is insufficient, the boundary sticks to the foundation and moves at the same velocity as the foundation. Condition (2.11) describes the so-called slip zone, i.e. when the tangential stress reaches its greatest value, the boundary does not move in tandem with the foundation. The scalar $\lambda \geqslant 0$ is a multiplier indicating the relative direction of the slip between the body and the foundation. We note that we can recover the classic version of Coulomb's law by taking $F=\mu p$, where $\mu$ is the coefficient of friction. However, this more general formulation allows us to consider coefficients of friction which may vary with the normal compliance, and therefore with a power of the surface contract traction.

Finally, we describe the boundary condition for the temperature on $\Gamma_{C}$. Since the power that is generated by the frictional contact forces is proportional to $\left|\sigma_{T}\right|\left|u_{T, t}-\phi\right|$, we take a boundary condition of the following form:

$$
k_{i j} \frac{\partial \theta}{\partial x_{i}} n_{j}=F\left(x, u_{n}-g\right) s_{c}\left(x,\left|v_{T}-\phi\right|\right)-k\left(\theta-\theta_{R}\right) \quad \text { on } \Gamma_{C} .
$$

Here $F$ is as before, $v_{T}=u_{T, t}$ is the surface velocity, $s_{c}(x, r)$ is a prescribed function, $k$ is the coefficient of heat exchange, and $\theta_{R}$ is the prescribed temperature of the foundation. The inclusion of a term such as this is required if the effects of frictional heating are to be taken into account, which is essential in many applications. For example, the sudden application of car brakes may lead to the dissipation of energy in the form of frictional heating at a rate of as much as $300 \mathrm{HP}$.

\section{Weak formulation and main result}

In this section we give a variational formulation of conditions (2.1)-(2.12), since it is well-known that in general, problems containing contact conditions do not have classical solutions. To this end we introduce the following notation.

$$
\begin{aligned}
& E=\left\{v \in\left(H^{1}(\Omega)\right)^{3} ; v=0 \text { on } \Gamma_{D}\right\}, \\
& V=\left\{\eta \in H^{1}(\Omega) ; \eta=0 \text { on } \Gamma_{D} \cup \Gamma_{N}\right\}, \\
& \mathbb{E}=L^{2}(0, T ; E) \text { and } \mathbb{V}=L^{2}(0, T ; V) .
\end{aligned}
$$


Below, we will use $|\cdot|$ to denote the norm of either $H^{3}=\left(L^{2}(\Omega)\right)^{3}$ or of $H=L^{2}(\Omega)$ and use $(\cdot, \cdot)_{H}$ or $(\cdot, \cdot)_{H}^{3}$ to denote the associated inner product. Similarly, we will use $(\xi, \eta)_{V}=\left(\xi_{x}, \eta_{x}\right)_{H}$ to denote the inner product on $V$, and $(v, w)_{E}=(\nabla v, \nabla w)_{H^{3}}$ to denote the inner product on $E$. We will use $\|\cdot\|$ to denote the associated norms in either $E$ or $V$, as the meaning will be clear from the context. These inner products and norms are equivalent to the usual inner products and norms on $E$ or $V$. Finally, we use $\langle\cdot, \cdot\rangle$ to denote the duality pairing between $\mathbb{E}$ and $\mathbb{E}^{\prime}$, or $\mathbb{V}$ and $\mathbb{V}^{\prime}$, as once again the meaning will be evident from the context.

We now describe the regularity assumptions on the data. We suppose that the coefficients of elasticity, viscosity, thermal expansion and thermal conductivity satisfy

$$
\begin{aligned}
a_{i j k l}, & b_{i j k l}, c_{i j}, k_{i j}, \in L^{\infty}(\Omega), \\
a_{i j k l} & =a_{j i k l} \quad a_{i j k l}=a_{k l i j} \quad a_{i j k l}=a_{i j l k}, \\
a_{i j k l} \chi_{i j} \chi_{k l} & \geqslant \alpha_{1} \chi_{i j} \chi_{i j} \quad \text { for all symmetric tensors } \chi_{i j}, \\
b_{i j k l} & =b_{j i k l} \quad b_{i j k l}=b_{k l i j} \quad b_{i j k l}=b_{i j l k}, \\
b_{i j k l} \chi_{i j} \chi_{k l} & \geqslant \alpha_{2} \chi_{i j} \chi_{i j} \quad \text { for all symmetric tensors } \chi_{i j}, \\
c_{i j} & =c_{j i} \quad k_{i j}=k_{j i}, \\
k_{i j} & \geqslant \alpha_{3} \delta_{i j} .
\end{aligned}
$$

Here $\alpha_{1}, \alpha_{2}, \alpha_{3}$ are fixed positive constants and $\delta_{i j}$ is the Kronecker $\delta$.

The gap function (in the reference configuration) is assumed to satisfy

$$
g \in L^{\infty}\left(\Gamma_{C}\right) \text {, }
$$

while the surface (tangential) velocity of the rigid foundation is taken as

$$
\phi \in\left(L^{\infty}\left(\Gamma_{C}\right)\right)^{3} .
$$

The functions related to contact, friction and heat generation satisfy the following conditions:

$$
\begin{aligned}
& p: \Omega \times R \rightarrow R^{+} \cup 0 \text { is Borel measurable and, } \\
& \quad \text { for each } x, p(x, \cdot) \text { is continuous, vanishes for } \\
& r<0 \text { and } p(x, r) \leqslant c+c|r|^{m_{n}} \text {. } \\
& F: \Omega \times R \rightarrow R^{+} \cup 0 \text { is Borel measurable and, for each } \\
& \quad x, F(x, \cdot) \text { is continuous, and } F(x, r) \leqslant c+c|r| . \\
& s_{c}: \Omega \times R \rightarrow R \text { is Borel measurable and, for each } \\
& \quad x, s_{c}(x, \cdot) \text { is continuous, and } s_{c}(\cdot, r) \leqslant c .
\end{aligned}
$$

We always assume that

$$
0 \leqslant m_{n}<2 .
$$

The above growth assumptions are made so that we can use the standard trace theorem $H^{1}(\Omega) \rightarrow L^{4}(\Gamma)[10]$ to conclude that if $w \in H^{1}(\Omega)$, then $p(\cdot, w(\cdot)-g) \in L^{2}\left(\Gamma_{C}\right)$, $F(\cdot, w(\cdot)-g) \in L^{4}\left(\Gamma_{C}\right)$, and $s_{c}(\cdot,|w(\cdot)-\phi(\cdot)|) \in L^{\infty}\left(\Gamma_{C}\right)$. We do not know if there is any physical significance to these restrictions on the growth of the functions $p, F$ and $s_{c}$. From the point of view of applications the growth conditions contained in (3.6) and (3.8) are 
not overly restrictive. Even the boundedness condition (3.7) is reasonable for applications which involve only small slip velocities. We remark that Kuttler [14] has shown that certain restrictions may be removed if $p$ is assumed to satisfy a lower growth estimate and if all thermal effects are ignored.

Next, we describe the regularity assumptions on the initial and boundary data:

$$
\begin{array}{cl}
u_{0} \in E, \quad v_{0} \in\left(L^{2}(\Omega)\right)^{3}, \quad \theta_{0} \in L^{2}(\Omega), \\
\Theta_{b} \in H^{1}(\Omega),\left.\quad \Theta_{b}\right|_{\Gamma_{D} \cup \Gamma_{N}}=\theta_{b} \quad \theta_{R} \in L^{2}\left(\Gamma_{C}\right), \quad f_{N} \in\left(L^{2}\left(\Gamma_{N}\right)\right)^{3} .
\end{array}
$$

Finally, the forces and volume heat source satisfy

$$
\begin{aligned}
f_{A} & \in \mathbb{E}^{\prime}, \\
q & \in \mathbb{V}^{\prime} .
\end{aligned}
$$

We can now give the weak, or variational, formulation of our problem. For technical reasons, it is convenient to shift the temperature function so that it is zero on $\Gamma_{D} \cup \Gamma_{N}$. For this purpose, we introduce the quantities $\xi=\theta-\Theta_{b}$ and $\xi_{0}=\theta_{0}-\Theta_{b}$.

Definition 3.1 A pair of functions $\{u, \xi\}$ is said to be a weak solution to (2.1)-(2.12) provided that:

$$
\begin{aligned}
u, u^{\prime} & \in \mathbb{E}, \quad u^{\prime \prime} \in \mathbb{E}^{\prime}, \quad u(\cdot, 0)=u_{0}, \quad\left(u^{\prime}\right)(\cdot, 0)=v_{0}, \\
\xi & \in \mathbb{V}, \quad \xi^{\prime} \in \mathbb{V}^{\prime}, \quad \xi(\cdot, 0)=\xi_{0},
\end{aligned}
$$

and for all $z$ such that $z, z^{\prime} \in \mathbb{E}$ we have that

$$
\begin{aligned}
\int_{0}^{T} & \left\langle u_{i}^{\prime \prime}, z_{i}-u_{i}\right\rangle+\int_{0}^{T} \int_{\Omega} a_{i j k l} u_{k, l}\left(z_{i, j}-u_{i, j}\right)+\int_{0}^{T} \int_{\Omega} b_{i j k l} u_{k, l}^{\prime}\left(z_{i, j}-u_{i, j}\right) \\
& -\int_{0}^{T} \int_{\Omega} c_{i j} \xi\left(z_{i, j}-u_{i, j}\right)+\int_{0}^{T} \int_{\Gamma_{C}} p\left(x, u_{n}-g\right)\left(z_{n}-u_{n}\right) d \Gamma d t \\
& +\int_{0}^{T} \int_{\Gamma_{C}} F\left(x, u_{n}-g\right)\left[\left|u_{T}^{\prime}-\phi+z_{T}\right|-\left|u_{T}^{\prime}-\phi\right|\right] d \Gamma d t \\
\geqslant & \int_{0}^{T}\left\langle f_{A i},\left(z_{i}-u_{i}\right)\right\rangle-\int_{0}^{T} \int_{\Omega} c_{i j} \Theta_{b}\left(z_{i, j}-u_{i, j}\right)+\int_{0}^{T} \int_{\Gamma_{N}} f_{N i}\left(z_{i}-u_{i}\right) d \Gamma d t,
\end{aligned}
$$

and for all $\eta \in \mathbb{V}$ we have that

$$
\begin{aligned}
& \int_{0}^{T} \int_{\Omega} \xi^{\prime} \eta+\int_{0}^{T} \int_{\Omega} k_{i j} \xi_{, i} \eta_{, j}+\int_{0}^{T} \int_{\Omega} c_{i j} u_{i, j}^{\prime} \eta \\
& \quad-\int_{0}^{T} \int_{\Gamma_{C}} F\left(x, u_{n}-g\right) s_{c}\left(x,\left|u_{T}^{\prime}-\phi\right|\right) \eta d \Gamma d t+\int_{0}^{T} \int_{\Gamma_{C}} k \xi \eta d \Gamma d t \\
& =\int_{0}^{T}\langle q, \eta\rangle+\int_{0}^{T} \int_{\Gamma_{C}} k\left(\Theta_{b}-\theta_{R}\right) \eta d \Gamma d t-\int_{0}^{T} \int_{\Omega} k_{i j} \Theta_{b, i} \eta_{, j} .
\end{aligned}
$$

Here the prime over a function represents the time derivative, and over a space its topological dual. Note that since $u, u^{\prime} \in \mathbb{E}$ we have that $u \in L^{\infty}\left(0, T ; H^{1}(\Omega)\right)$ and so 
$F\left(\cdot, u_{n}-g\right) \in L^{\infty}\left(0, T ; L^{4}\left(\Gamma_{C}\right)\right)$. Thus the te containing both $F$ and $s_{c}$ in $(3.15)$ is welldefined. We now define a number of operators which will be needed to write the problem in an abstract operator form. Let

$$
\begin{aligned}
& D, C, P: E \rightarrow E^{\prime} \\
& M_{1}: E \rightarrow V^{\prime} \\
& H, K: V \rightarrow V^{\prime} \\
& M_{2}: V \rightarrow E^{\prime} \\
& S: E \times E \rightarrow V^{\prime}
\end{aligned}
$$

be defined by

$$
\begin{gathered}
\langle D u, v\rangle=\int_{\Omega} a_{i j k l} u_{k, l} v_{i, j}, \\
\langle C u, v\rangle=\int_{\Omega} b_{i j k l} u_{k, l} v_{i, j}, \\
\langle H \xi, \eta\rangle=\int_{\Gamma_{C}} k \xi \eta d \Gamma, \\
\langle K \xi, \eta\rangle=\int_{\Omega} k_{i j} \xi_{, i} \eta, j, \\
\left\langle M_{1} v, \eta\right\rangle=\int_{\Omega} c_{i j} v_{i, j} \eta, \\
\left\langle M_{2} \xi, v\right\rangle=-\int_{\Omega} c_{i j} \xi v_{i, j}, \\
\langle P u, v\rangle=\int_{\Gamma_{C}} p\left(x, u_{n}-g\right) v_{n} d \Gamma, \\
\langle S(u, v), \eta\rangle=-\int_{\Gamma_{C}} F\left(x, u_{n}-g\right) s_{c}\left(x,\left|v_{T}-\phi\right|\right) \eta d \Gamma .
\end{gathered}
$$

We note that, with one exception, each of the above operators extends to an operator defined on the obviously corresponding space of square-integrable vector-valued functions in a natural way. For example, $D$ extends to an operator from $\mathbb{E}$ to $\mathbb{E}^{\prime}$ by $(D u)(t)=$ $D(u(t))$. In the case of the operator $S$, however, the desired extension has the action $L^{\infty}(0, T ; E) \times \mathbb{E} \rightarrow \mathbb{V}^{\prime}$ and not $\mathbb{E} \times \mathbb{E} \rightarrow \mathbb{V}^{\prime}$. In an abuse of notation, we will use the same letter below to denote both the original operator and its extension, since the meaning will be clear from the context. We can now formulate the problem abstractly as follows.

Find $(u, \xi)$ satisfying (3.13) and such that:

$$
\begin{gathered}
\xi^{\prime}+K \xi+M_{1} u^{\prime}+S\left(u, u^{\prime}\right)+H \xi=Q, \text { in } \mathbb{V}^{\prime}, \\
u^{\prime \prime}+C u^{\prime}+D u+P u+\partial_{2} j\left(u, u^{\prime}\right)+M_{2} \xi \ni f, \text { in } \mathbb{E}^{\prime} .
\end{gathered}
$$

Here $f$ and $Q$ are given by

$$
\langle f, w\rangle_{\mathbb{E}^{\prime}, \mathbb{E}}=\int_{0}^{T}\left\langle f_{A i}, w_{i}\right\rangle-\int_{0}^{T} \int_{\Omega} c_{i j} \Theta_{b} w_{i, j}+\int_{0}^{T} \int_{\Gamma_{N}} f_{N_{i}} w_{i} d \Gamma d t,
$$


and

$$
\langle Q, \eta\rangle_{\mathbb{V}^{\prime}, \mathbb{V}}=\int_{0}^{T}\langle q, \eta\rangle+\int_{0}^{T} \int_{\Gamma_{C}} k\left(\Theta_{b}-\theta_{R}\right) \eta d \Gamma d t-\int_{0}^{T} \int_{\Omega} k_{i j} \Theta_{b, i} \eta_{j},
$$

and $\partial_{2} j$ denotes the partial subdifferential with respect to the second argument of

$$
j(u, v)=\int_{0}^{T} \int_{\Gamma_{C}} F\left(x, u_{n}-g\right)\left|v_{T}-\phi\right| d \Gamma d t .
$$

Note that $j$ is convex with respect to that argument.

The main result in this paper is

Theorem 3.2 Assume that (3.1)-(3.12) hold. Then problem (3.24)-(3.25) has a solution.

We conclude that the problem (2.1)-(2.12) possesses a weak solution in the sense of Definition 3.1

The proof of the theorem will be given in $\S 5$, where the solution is obtained as a limit of solutions to a sequence of approximate problems that are presented in the next section.

\section{The approximate problem}

In this section we consider a sequence of regularized problems which approximate our original problem. The solution to these problems is obtained by the application of an abstract existence theorem for first order evolution equations that is a special case of results contained in Kuttler [18] (see also Kuttler [19]). To apply this result, we introduce the following spaces and operators.

Let $F, G$ be reflexive Banach spaces such that $F \subseteq G,\|\|_{F} \geqslant\|\|_{G}$ and $F$ is dense in $G$. Thus we may write $F \subseteq G \subseteq G^{\prime} \subseteq F^{\prime}$. Suppose that $B \in L\left(G, G^{\prime}\right)$ is positive and symmetric, i.e.

$$
\begin{gathered}
\langle B g, g\rangle \geqslant 0, \\
\langle B g, h\rangle=\langle B h, g\rangle
\end{gathered}
$$

Let $\mathbb{F}=L^{2}(a, b ; F)$ and define $\mathbb{X}=\left\{w \in \mathbb{F}:(B w)^{\prime} \in \mathbb{F}^{\prime}\right\}$ with norm given by $\|w\|_{\mathbb{X}}=$ $\|w\|_{\mathbb{F}}+\left\|(B w)^{\prime}\right\|_{\mathbb{F}^{\prime}}$. Here the differentiation is taken in the sense of $F^{\prime}$ valued distributions. It is easy to see that $\mathbb{X}$ is also a reflexive Banach space. We suppose further that $A: \mathbb{F} \rightarrow \mathbb{F}^{\prime}$ is an operator of the form $A w(t)=\mathscr{A}(t, w(t))$, where the operators $\mathscr{A}(t, \cdot): F \rightarrow F^{\prime}$ and for which the following conditions hold:

$$
\begin{gathered}
A: \mathbb{X} \rightarrow \mathbb{X}^{\prime} \text { is pseudomonotone [20], } \\
A: \mathbb{F} \rightarrow \mathbb{F}^{\prime} \text { is bounded }
\end{gathered}
$$

and, for some $\lambda$,

$$
\lim _{\|w\|_{\mathbb{F}} \rightarrow \infty}\left(\|w\|_{\mathbb{F}}\right)^{-1}\left(\lambda\langle B w, w\rangle_{\mathbb{G}, \mathbb{G}^{\prime}}+\langle A w, w\rangle_{\mathbb{F}, \mathbb{F}^{\prime}}\right)=\infty .
$$

The following existence result is a special case of results in Kuttler [18].

Theorem 4.1 Let $A$ and $B$ be as described above. Then for each $w_{0} \in G$ and $l \in \mathbb{F}^{\prime}$ there 
exists $a w \in \mathbb{X}$, satisfying

$$
\begin{aligned}
(B w)^{\prime}+A w & =l \text { in } \mathbb{F}^{\prime} \\
B w(0) & =B w_{0} \text { in } G^{\prime}
\end{aligned}
$$

We turn next to describe the regularized problem whose solution will be given by Theorem 4.1. For this purpose we need the truncation of the operator $P$ given by

$$
\left\langle P^{h} u, v\right\rangle=\int_{\Gamma_{C}} p^{h}\left(x, u_{n}-g\right) v_{n} d \Gamma
$$

where

$$
p^{h}(x, r)= \begin{cases}p(x, r), & r \leqslant 1 / h \\ p(x, 1 / h) & r>1 / h\end{cases}
$$

To replace the inclusion in (3.25) with equality we employ a regularization of the norm function. Let $\left\{\psi^{h}(\cdot)\right\}_{h>0}$ be a family of smooth approximations to $|\cdot|$, such that, for each $h>0, \psi^{h} \in C^{1}\left(\mathbb{R}^{m}\right)$ is positive and convex, $\left|\nabla \psi^{h}\right| \leqslant 2$ and, for all $s \in \mathbb{R}^{m}, 0 \leqslant\left\langle\nabla \psi^{h}(s), s\right\rangle$ and $\left|\psi^{h}(s)-\right| s|| \leqslant h$. We define the operator $F^{h}: E \times E \rightarrow E^{\prime}$ by

$$
\left\langle F^{h}(u, v), w\right\rangle=\int_{\Gamma_{C}} F\left(x, u_{n}-g\right) \nabla \psi^{h}\left(v_{T}-\phi\right) \cdot w_{T} d \Gamma .
$$

Finally, we let $R: E \rightarrow E^{\prime}$ be the Riesz map.

We now consider the following regularized problem:

Find $(u, v, \xi)$ satisfying

$$
\begin{aligned}
\xi \in \mathbb{V} \quad \xi^{\prime} \in \mathbb{V}^{\prime} \quad u, u^{\prime}, v \in \mathbb{E} \quad v^{\prime} \in \mathbb{E}^{\prime} ; \\
\xi^{\prime}+K \xi+M_{1} v+S(u, v)+H \xi=Q, \text { in } \mathbb{V}^{\prime}, \\
v^{\prime}+C v+D u+P^{h} u+F^{h}(u, v)+M_{2} \xi=f, \text { in } \mathbb{E}^{\prime}, \\
(R u)^{\prime}-R v=0, \text { in } \mathbb{E}^{\prime},
\end{aligned}
$$

together with the initial conditions

$$
\begin{array}{ll}
u(0)=u_{0}, & u_{0} \in E, \\
\xi(0)=\xi_{0}, & \xi_{0} \in L^{2}(\Omega), \\
v(0)=v_{0}, & v_{0} \in\left(L^{2}(\Omega)\right)^{3} .
\end{array}
$$

We have the following existence result for this problem.

Theorem 4.2 Let $h>0$. Then there exists a solution to problem (4.8)-(4.14).

Proof We fit the problem into the framework of Theorem 4.1 by taking $F=V \times E \times E$ and $G=H \times H^{3} \times E$. Let $B: G \rightarrow G^{\prime}$ be given by

$$
B\left(\begin{array}{l}
\xi \\
v \\
u
\end{array}\right)=\left(\begin{array}{c}
\xi \\
v \\
R u
\end{array}\right)
$$


and let $\mathscr{A}(t, \cdot): F \rightarrow F^{\prime}$ be given by

$$
\mathscr{A}\left(t,\left(\begin{array}{l}
\xi \\
v \\
u
\end{array}\right)\right)=\left(\begin{array}{c}
K \xi+M_{1} v+H \xi+S(u, v) \\
C v+D u+P^{h} u+F^{h}(u, v)+M_{2} \xi \\
-R v
\end{array}\right) .
$$

Finally, we let

$$
l=\left(\begin{array}{l}
q \\
f \\
0
\end{array}\right)
$$

and

$$
w_{0}=\left(\begin{array}{c}
\xi_{0} \\
v_{0} \\
R u_{0}
\end{array}\right) .
$$

Now the verification of (4.1)-(4.5) for this setting is largely routine but we would like to single out two items for special mention. In checking the pseudomonotonicity condition (4.3) we use the fact that $\mathscr{A}=\mathscr{A}_{1}+\mathscr{A}_{2}$, where

$$
\mathscr{A}_{1}\left(t,\left(\begin{array}{l}
\xi \\
v \\
u
\end{array}\right)\right)=\left(\begin{array}{c}
K \xi+M_{1} v+H \xi \\
C v+D u+M_{2} \xi \\
-R v
\end{array}\right)
$$

gives rise to a bounded linear operator from $\mathbb{X}$ to $\mathbb{X}^{\prime}$ (hence weakly continuous and thus pseudomonotone [20]) and

$$
\mathscr{A}_{2}\left(t,\left(\begin{array}{l}
\xi \\
v \\
u
\end{array}\right)\right)=\left(\begin{array}{c}
S(u, v) \\
P^{h} u+F^{h}(u, v) \\
0
\end{array}\right)
$$

is a weak to norm continuous operator from $\mathbb{X}$ to $\mathbb{X}^{\prime}$. To establish the latter property the following lemma is employed.

Lemma 4.3 Let $\mathbb{Y}=\left\{u \in \mathbb{E}:(R u)^{\prime} \in \mathbb{E}^{\prime}\right\}$ with norm given by $\|u\|_{\mathbb{Y}}=\|u\|_{\mathbb{E}}+\left\|(R u)^{\prime}\right\|_{\mathbb{E}^{\prime}}$ and let $P^{h}: \mathbb{Y} \rightarrow \mathbb{Y}^{\prime}, F^{h}: \mathbb{Y} \times \mathbb{Y} \rightarrow \mathbb{Y}^{\prime}$ and $S: \mathbb{Y} \times \mathbb{Y} \rightarrow \mathbb{Y}^{\prime}$ be given by (4.6),(4.7) and (3.23), respectively. Then each of these operators is weak-to-norm continuous.

Proof We establish the result only for the operator $P^{h}$ since the proofs for $F^{h}$ and $S$ are similar. To establish this result it suffices to show that every subsequence of a weakly convergent sequence $u_{m}$ has a further subsequence whose images under $P^{h}$ converge to $P^{h} u$. So let $u_{m_{j}}$ be a subsequence of $u_{m}$. Since $u_{m_{j}}$ is bounded in $\mathbb{Y}$ and since the injection $\left(H^{1}(\Omega)\right)^{3} \rightarrow\left(H^{1-\epsilon}(\Omega)\right)^{3}$ is compact for any $\epsilon>0$ [20], we have by a theorem of Lions [21], that $u_{m_{j}}$ is relatively compact in $L^{2}\left(0, T ;\left(H^{1-\epsilon}(\Omega)\right)^{3}\right)$. By the continuity of the trace map $L^{2}\left(0, T ;\left(H^{1-\epsilon}(\Omega)\right)^{3}\right) \rightarrow L^{2}\left(0, T ;\left(L^{2}\left(\Gamma_{C}\right)\right)^{3}\right)=\left(L^{2}\left((0, T) \times \Gamma_{C}\right)\right)^{3}[10]$, we may, upon passing to a subsequence, suppose that $u_{m_{j}}(x, t) \rightarrow u(x, t)$ for almost all $(x, t) \in \Gamma_{C} \times(0, T)$. Since $p$ and hence $p^{h}$ is continuous in the second variable, we have that $p^{h}\left(x,\left(u_{m_{j}}\right)_{n}(x, t)-g\right) \rightarrow$ $p^{h}\left(x, u_{n}(x, t)-g\right)$, for almost all $(x, t) \in \Gamma_{C} \times(0, T)$. Since $\left|p^{h}\left(x,\left(u_{m_{j}}\right)_{n}(x, t)-g\right)\right|^{2+\epsilon} \leqslant c_{1}+$ $c_{2}\left|\left(u_{m_{j}}\right)_{n}(x, t)-g\right|^{(2+\epsilon) m_{n}} \leqslant c_{1}+c_{2}\left|\left(u_{m_{j}}\right)_{n}(x, t)-g\right|^{4}$ for $\epsilon$ sufficiently small, it follows from the 
continuity of the trace map $\mathbb{E} \rightarrow L^{2}\left(0, T ;\left(L^{4}\left(\Gamma_{C}\right)\right)^{3}\right)$ that the sequence $p^{h}\left(\cdot,\left(u_{m_{j}}\right)_{n}(\cdot, \cdot)-g\right)$ is bounded in $L^{2+\epsilon}\left(\Gamma_{C} \times(0, T)\right)$. Consequently, $p^{h}\left(\cdot,\left(u_{m_{j}}\right)_{n}(\cdot, \cdot)-g\right)$ converges in $L^{2}\left(\Gamma_{C} \times\right.$ $(0, T))$ to $p^{h}\left(\cdot, u_{n}(\cdot, \cdot)-g\right)$. Thus, we have that, for any $v \in \mathbb{Y},\left|\left\langle P^{h} u_{m_{j}}-P^{h} u, v\right\rangle_{\mathbb{Y}^{\prime}, \mathbb{Y}}\right| \leqslant$ $\left\|\left(p^{h}\left(\cdot,\left(u_{m_{j}}\right)_{n}(\cdot, \cdot)-g\right)-p^{h}\left(\cdot, u_{n}(\cdot, \cdot)-g\right)\right)\right\|_{L^{2}\left(\Gamma_{C} \times(0, T)\right)}\left\|v_{n}(\cdot, \cdot)\right\|_{L^{2}\left(\Gamma_{C} \times(0, T)\right)} \rightarrow 0$ as $j \rightarrow \infty$. This gives the result.

In checking the coercivity condition (4.5) we estimate terms using Hölder's inequality, the standard trace theorem $H^{1}(\Omega) \rightarrow L^{4}\left(\Gamma_{C}\right)$, and the first two conclusions of the following technical result. This result follows from Korn's inequalities [22] and conditions (3.1)-(3.2).

Lemma 4.4 Assume that (3.1)-(3.2) hold. Then there exist positive constants $\alpha$ and $\beta$ such that

$$
\begin{aligned}
\langle C v, v\rangle & \geqslant \alpha|| v \|_{E}^{2}-\beta|v|_{H^{3}}^{2}, \\
\langle K \xi, \xi\rangle & \geqslant \alpha\|\xi\|_{V}^{2}-\beta|\xi|_{H}^{2}, \\
\langle D u, u\rangle & \geqslant \alpha|| u \|_{E}^{2}-\beta|u|_{H^{3}}^{2} .
\end{aligned}
$$

We also estimate the term containing $F^{h}$ in the following way:

$$
\begin{aligned}
\left\langle F^{h}(u, v), v\right\rangle_{\mathbb{Y}^{\prime}, \mathbb{Y}}= & \int_{0}^{t} \int_{\Gamma_{C}} F\left(x, u_{n}-g\right)\left(\nabla \psi^{h}\right)\left(v_{T}-\phi\right) \cdot v_{T} d \Gamma d s \\
= & \int_{0}^{t} \int_{\Gamma_{C}} F\left(x, u_{n}-g\right)\left(\nabla \psi^{h}\right)\left(v_{T}-\phi\right) \cdot\left(v_{T}-\phi\right) d \Gamma d s \\
& \quad \quad \quad \int_{0}^{t} \int_{\Gamma_{C}} F\left(x, u_{n}-g\right)\left(\nabla \psi^{h}\right)\left(v_{T}-\phi\right) \cdot \phi d \Gamma d s \\
\geqslant & -c-c\|u\|_{\mathbb{E}}^{2},
\end{aligned}
$$

where $c$ is a positive constant that depends only on the data. This completes our discussion of how (4.5) is established and concludes the proof of Theorem 4.2.

We turn next to obtain an a priori estimate on the solutions to the approximate problem.

Theorem 4.5 Let $\left(u_{h}, v_{h}, \xi_{h}\right)$ be a solution to problem (4.8)-(4.14) corresponding to the parameter $h$. Then there exists a positive constant $C$ that is independent of $h$ such that, for all $t$ in $0 \leqslant t \leqslant T$,

$$
\left|v_{h}(t)\right|^{2}+\left\|u_{h}(t)\right\|^{2}+\int_{0}^{t}\left\|\left.v_{h}(s)\right|^{2} d s+\left|\xi_{h}(t)\right|^{2}+\int_{0}^{t}\right\| \xi_{h}(s) \|^{2} d s \leqslant C .
$$

Proof Let $c$ below represent a positive constant whose value may change from line to line, but in all cases depends only on the data and coefficients of the approximate problem. We will also omit the subscript $h$ below for the sake of simplicity. We begin by letting (4.10) act on $v$. Now, Theorem 1(2) of Kuttler [18] and our choice of $F, G$ and $B$ implies that $\left\langle v^{\prime}, v\right\rangle_{E^{\prime}, E}(t)=(1 / 2)(d / d t)|v(t)|_{H}^{2}$. Using this fact and Cauchy's inequality with 
$\epsilon$, we obtain

$$
\begin{aligned}
& \frac{1}{2}|v(t)|^{2}+\int_{0}^{t}\langle D u, v\rangle d s+\int_{0}^{t}\langle C v, v\rangle d s \\
& \quad+\int_{0}^{t}\left\langle P^{h}(u), v\right\rangle d s+\int_{0}^{t}\left\langle F^{h}(u, v), v\right\rangle d s+\int_{0}^{t}\left\langle M_{2} \xi, v\right\rangle d s \\
& \leqslant c+\int_{0}^{t}\langle f, v\rangle d s .
\end{aligned}
$$

We will now estimate the terms containing the integrals in (4.17). Using Lemma 4.4 and (4.11), it follows that the first integral in (4.17) is equal to

$$
\begin{aligned}
\int_{0}^{t}\langle D u, v\rangle d s & =\frac{1}{2}\langle D u(t), u(t)\rangle-\frac{1}{2}\left\langle D u_{0}, u_{0}\right\rangle \\
& \geqslant-c+\frac{1}{2} \alpha\|u(t)\|^{2}-\frac{1}{2} \beta|u(t)|^{2} \\
& \geqslant-c+\frac{1}{2} \alpha\|u(t)\|^{2}-c \int_{0}^{t}|v(s)|^{2} d s
\end{aligned}
$$

Another application of Lemma 4.4 yields

$$
\int_{0}^{t}\langle C v, v\rangle d s \geqslant \alpha \int_{0}^{t}\|v(s)\|^{2} d s-\beta \int_{0}^{t}|v(s)|^{2} d s .
$$

To estimate the term involving $P^{h}$ we observe that, for $u \in \mathbb{E}$ with $u^{\prime}=v \in \mathbb{E}$,

$$
\begin{aligned}
\frac{d}{d t} \int_{\Gamma_{C}} \int_{g}^{u_{n}} p^{h}(x, r-g) d r d \Gamma & =\int_{\Gamma_{C}} \frac{\partial}{\partial t} \int_{g}^{u_{n}} p^{h}(x, r-g) d r d \Gamma \\
& =\int_{\Gamma_{C}} p^{h}\left(x, u_{n}-g\right) \frac{\partial u_{n}}{\partial t} d \Gamma \\
& =\int_{\Gamma_{C}} p^{h}\left(x, u_{n}-g\right) v_{n} d \Gamma \\
& =\left\langle P^{h}(u), v\right\rangle .
\end{aligned}
$$

It follows that

$$
\begin{aligned}
& \int_{0}^{t}\left\langle P^{h}(u), v\right\rangle d s= \int_{\Gamma_{C}} \int_{g}^{u_{n}(t)} p^{h}(x, r-g) d r d \Gamma \\
& \quad-\int_{\Gamma_{C}} \int_{g}^{u_{n}(0)} p^{h}(x, r-g) d r d \Gamma \\
& \geqslant-c,
\end{aligned}
$$

where we have used the fact that when $u_{n}(t) \geqslant g$, then $p^{h}(x, r-g) \geqslant 0$ and when $u_{n}(t)<g$, $p^{h}(x, r-g)=0$. The estimate for the term containing $F^{h}$ is identical with (4.16):

$$
\int_{0}^{t} \int_{\Gamma_{C}} F\left(x, u_{n}-g\right)\left(\nabla \psi^{h}\right)\left(v_{T}-\phi\right) \cdot v_{T} d \Gamma d s \geqslant-c-c\|u\|_{\mathbb{E}}^{2} .
$$

The integrals containing $M_{2}$ and $f$ are estimated simply by using Cauchy's inequality 
with $\epsilon$ :

$$
\begin{gathered}
\left|\int_{0}^{t}\left\langle M_{2} \xi, v\right\rangle d s\right| \leqslant c \int_{0}^{t}|\xi(s)|^{2} d s+\frac{1}{8} \alpha \int_{0}^{t}\|v(s)\|^{2} d s, \\
\left|\int_{0}^{t}\langle f, v\rangle d s\right| \leqslant c+\frac{1}{8} \alpha \int_{0}^{t}\|v(s)\|^{2} d s .
\end{gathered}
$$

Combining the estimates (4.17)-(4.24) yields

$$
\begin{aligned}
& |v(t)|^{2}+\|u(t)\|^{2}+\int_{0}^{t}\|v(s)\|^{2} d s \\
& \quad \leqslant c\left(1+\int_{0}^{t}|v(s)|^{2} d s+\int_{0}^{t}|\xi(s)|^{2} d s+\int_{0}^{t}\|u(s)\|^{2} d s\right) .
\end{aligned}
$$

Now we apply Gronwall's inequality and obtain

$$
|v(t)|^{2}+\|u(t)\|^{2}+\int_{0}^{t}\|v(s)\|^{2} d s \leqslant c+c \int_{0}^{t}|\xi(s)|^{2} d s .
$$

We leave (4.25) for the moment and turn to the energy equation (4.9). We let (4.9) act on $\xi$ and estimate terms as above to obtain

$$
\begin{aligned}
& \frac{1}{2}|\xi(t)|^{2}+\alpha \int_{0}^{t}\|\xi(s)\|^{2} d s+\int_{0}^{t} \int_{\Gamma_{C}} k|\xi|^{2} d \Gamma d s \\
& \quad \leqslant c+c \int_{0}^{t}|\xi(s)|^{2} d s+c \int_{0}^{t}\|v(s)\|^{2} d s-\int_{0}^{t}\langle S(u, v), \xi\rangle d s .
\end{aligned}
$$

We focus on the last integral. Using (3.6), (3.7), (3.23) and Cauchy's inequality with $\epsilon$, we obtain

$$
\begin{aligned}
\left|\int_{0}^{t}\langle S(u, v), \xi\rangle d s\right| & \leqslant c \int_{0}^{t} \int_{\Gamma_{C}}\left(1+\left|u_{n}-g\right|\right)|\xi| d \Gamma d s \\
& \leqslant c+\frac{\alpha}{2} \int_{0}^{t} \int_{\Gamma_{C}}|\xi(s)|^{2} d \Gamma d s+c \int_{0}^{t} \int_{\Gamma_{C}}\left|u_{n}-g\right|^{2} d \Gamma d s \\
& \leqslant c+\frac{\alpha}{2} \int_{0}^{t}\|\xi(s)\|^{2} d s+c \int_{0}^{t}\|u\|^{2} d s .
\end{aligned}
$$

Using the estimates (4.25) and (4.27) in (4.26) we obtain

$$
\begin{aligned}
& \frac{1}{2}|\xi(t)|^{2}+\frac{\alpha}{2} \int_{0}^{t}\|\xi(s)\|^{2} d s+\int_{0}^{t} \int_{\Gamma_{C}} k|\xi|^{2} d \Gamma d s \\
& \leqslant c+c \int_{0}^{t}|\xi(s)|^{2} d s .
\end{aligned}
$$

An application of Gronwall's inequality now yields

$$
|\xi(t)|^{2}+\int_{0}^{t}\|\xi(s)\|^{2} d s \leqslant c .
$$

Combining (4.29) and (4.25) leads to the desired conclusion. 


\section{Existence}

In this section we prove existence of a solution to problem (3.24)-(3.25) as a limit of a sequence of solutions to the regularized problem (4.9)-(4.11). It follows from Theorem 4.5 that for a given set of initial conditions the family of solutions $\left(u_{h}, v_{h}, \xi_{h}\right)$ is bounded in $\mathbb{E} \times \mathbb{E} \times \mathbb{V}$. From this and (4.9)-(4.11) it follows that $\left(R u_{h}^{\prime}, v_{h}^{\prime}, \xi_{h}^{\prime}\right)$ is bounded in $\mathbb{E}^{\prime} \times \mathbb{E}^{\prime} \times \mathbb{V}^{\prime}$. Consequently, there exists a weak limit point $(u, v, \xi) \in \mathbb{E} \times \mathbb{E} \times \mathbb{V}$ and a subsequence of parameters $h_{l}$ such that $h_{l} \rightarrow 0$ as $l \rightarrow \infty$ and such that the following types of convergence take place:

$$
\begin{aligned}
u_{l} \rightarrow u & \text { weakly in } \mathbb{E}, \\
v_{l} \rightarrow v & \text { weakly in } \mathbb{E}, \\
\xi_{l} \rightarrow \xi & \text { weakly in } \mathbb{V}, \\
\xi_{l}^{\prime} \rightarrow \xi^{\prime} & \text { weakly in } \mathbb{V}^{\prime}, \\
v_{l}^{\prime} \rightarrow v^{\prime} & \text { weakly in } \mathbb{E}^{\prime}, \\
R u_{l}^{\prime} \rightarrow R u^{\prime} & \text { weakly in } \mathbb{E}^{\prime} .
\end{aligned}
$$

With these results and Lemma 4.3 we may obviously pass to the limit in (4.9) and (4.10) in all terms except those involving $P^{h_{l}}$ and $F^{h_{l}}$. For the term involving $P^{h_{l}}$, the proof of Lemma 4.3 can be modified to show that $P^{h_{l}} u_{l} \rightarrow P u$ strongly in $\mathbb{E}^{\prime}$. For the term involving $F^{h_{l}}$ we may suppose, by passing to a subsequence, that, for some $\zeta \in \mathbb{E}^{\prime}$, $F^{h}\left(u_{l}, v_{l}\right) \rightarrow \zeta$ weakly in $\mathbb{E}^{\prime}$. It only remains to show that $\zeta \in \partial_{2}\left(u, u^{\prime}\right)$. Toward that end, we note that $F\left(\cdot, u_{l n}-g\right) \rightarrow F\left(\cdot, u_{n}-g\right)$ in $L^{4 / 3}\left(\Gamma_{C} \times(0, T)\right)$ and, for any $w \in \mathbb{E}$, $\psi^{h_{l}}\left(v_{l T}-\phi+w_{T}\right) \rightarrow\left|v_{T}-\phi+w_{T}\right|$ in $L^{4}\left(\Gamma_{C} \times(0, T)\right)$. Fix such a $w \in \mathbb{E}$. Then it follows from the convexity of $\psi^{h}$, the positivity of $F$ and Hölder's inequality that

$$
\begin{aligned}
\langle\zeta, w\rangle & =\lim _{l \rightarrow \infty}\left\langle F^{h}\left(u_{l}, v_{l}\right), w\right\rangle \\
& =\lim _{l \rightarrow \infty} \int_{0}^{T} \int_{\Gamma_{C}} F\left(u_{l n}-g\right) \nabla \psi^{h}\left(v_{l T}-\phi\right) \cdot w_{T} d \Gamma d t \\
& \leqslant \lim _{l \rightarrow \infty} \int_{0}^{T} \int_{\Gamma_{C}} F\left(u_{h n}-g\right)\left[\psi^{h}\left(v_{l T}-\phi+w_{T}\right)-\psi^{h}\left(v_{l T}-\phi\right)\right] d \Gamma d t \\
& \leqslant \int_{0}^{T} \int_{\Gamma_{C}} F\left(u_{n}-g\right)\left[\left|v_{T}-\phi+w_{T}\right|-\left|v_{T}-\phi\right|\right] d \Gamma d t .
\end{aligned}
$$

This completes the proof of Theorem 3.2.

\section{Uniqueness}

In this section we will prove a uniqueness result under additional assumptions on the data..

Theorem 6.1 Suppose that $p, F$ and $s_{c}$ satisfy the additional Lipschitz conditions

$$
\begin{aligned}
\left|p\left(x, r_{2}\right)-p\left(x, r_{1}\right)\right| & \leqslant K\left|r_{2}-r_{1}\right|\left(1+\left|r_{2}\right|^{2}+\left|r_{1}\right|^{2}\right) \\
\left|F\left(x, r_{2}\right)-F\left(x, r_{1}\right)\right| & \leqslant K\left|r_{2}-r_{1}\right|\left(1+\left|r_{2}\right|^{2}+\left|r_{1}\right|^{2}\right) \\
\left|s_{c}\left(x, r_{2}\right)-s_{c}\left(x, r_{1}\right)\right| & \leqslant K\left|r_{2}-r_{1}\right|,
\end{aligned}
$$

where $K$ is a constant. Then the problem (3.24)-(3.25) admits a unique solution. 
Proof Once again we let $c$ below denote a constant whose value may change from line to line. Let $\left(u_{1}, \xi_{1}\right)$ and $\left(u_{2}, \xi_{2}\right)$ be two solutions to problem (3.24)-(3.25) corresponding to the same initial data. We substitute $u_{1}$ into (3.25) and let the resulting expression act on $u_{1}^{\prime}-u_{2}^{\prime}$ and then substitute $u_{2}$ for $u$ in (3.25) and let the resulting expression act on $u_{2}^{\prime}-u_{1}^{\prime}$. Adding the two inequalities yields

$$
\begin{aligned}
& \frac{1}{2}\left|u_{1}^{\prime}(t)-u_{2}^{\prime}(t)\right|_{H^{3}}^{2}+\int_{0}^{t}\left\langle C\left(u_{1}^{\prime}-u_{2}^{\prime}\right), u_{1}^{\prime}-u_{2}^{\prime}\right\rangle d s+\int_{0}^{t}\left\langle D\left(u_{1}-u_{2}\right), u_{1}^{\prime}-u_{2}^{\prime}\right\rangle d s \\
& \quad+\int_{0}^{t}\left\langle P u_{1}-P u_{2}, u_{1}^{\prime}-u_{2}^{\prime}\right\rangle d s+\int_{0}^{t}\left\langle M_{2}\left(\xi_{1}-\xi_{2}\right), u_{1}^{\prime}-u_{2}^{\prime}\right\rangle d s \\
& \leqslant \int_{0}^{t} \int_{\Gamma_{C}}\left[F\left(x, u_{2 n}-g\right)-F\left(x, u_{1 n}-g\right)\right]\left[\left|u_{1 T}^{\prime}-\phi\right|-\left|u_{2 T}^{\prime}-\phi\right|\right] d \Gamma d s .
\end{aligned}
$$

We now estimate the various terms in (6.2). For the term involving $C$ we use the Korn inequality estimate (4.15):

$$
\int_{0}^{t}\left\langle C\left(u_{1}^{\prime}-u_{2}^{\prime}\right), u_{1}^{\prime}-u_{2}^{\prime}\right\rangle d s \geqslant \int_{0}^{t}\left(\alpha|| u_{1}^{\prime}-u_{2}^{\prime} \|_{E}^{2}-\beta\left|u_{1}^{\prime}-u_{2}^{\prime}\right|_{H^{3}}^{2}\right) d s .
$$

The term involving $D$ is evaluated as in (4.18):

$$
\begin{aligned}
\int_{0}^{t}\left\langle D\left(u_{1}-u_{2}\right), u_{1}^{\prime}-u_{2}^{\prime}\right\rangle d s & =\frac{1}{2}\left\langle D\left(u_{1}-u_{2}\right),\left(u_{1}-u_{2}\right)\right\rangle \\
& \geqslant \frac{1}{2} \alpha|| u_{1}(t)-u_{2}(t) \|_{E}^{2}-\frac{1}{2} \beta\left|u_{1}(t)-u_{2}(t)\right|_{H^{3}}^{2} .
\end{aligned}
$$

For the term involving $P$ we use (6.1) and the multi-Hölder inequality to obtain

$$
\begin{aligned}
& \left|\int_{0}^{t}\left\langle P u_{1}-P u_{2}, u_{1}^{\prime}-u_{2}^{\prime}\right\rangle d s\right| \\
& \quad \leqslant c \int_{0}^{t} \int_{\Gamma_{C}}\left|u_{1 n}-u_{2 n}\right|\left(1+\left|u_{1 n}-g\right|^{2}+\left|u_{2 n}-g\right|^{2}\right)\left|u_{1 n}^{\prime}-u_{2 n}^{\prime}\right| d \Gamma d s \\
& \quad \leqslant c \int_{0}^{t}\left\|u_{1}-u_{2}\left|\left\|_{E}|| u_{1}^{\prime}-u_{2}^{\prime}\right\|_{E} d s\right.\right. \\
& \quad \leqslant \frac{\alpha}{4} \int_{0}^{t}\left\|u_{1}^{\prime}-u_{2}^{\prime}\right\|_{E}^{2}+c \int_{0}^{t}\left\|u_{1}-u_{2}\right\|_{E}^{2} d s .
\end{aligned}
$$

The term involving $M_{2}$ is estimated simply by Cauchy's inequality with $\epsilon$ :

$$
\int_{0}^{t}\left\langle M_{2}\left(\xi_{1}-\xi_{2}\right), u_{1}^{\prime}-u_{2}^{\prime}\right\rangle d s \leqslant c \int_{0}^{t}\left|\xi_{1}-\xi_{2}\right|_{H}^{2} d s+\frac{\alpha}{4} \int_{0}^{t}|| u_{1}^{\prime}-\left.u_{2}^{\prime}\right|_{E} ^{2} d s .
$$

Finally, for the term involving $F$ we use (6.1) and the multi-Hölder inequality once again to obtain

$$
\begin{aligned}
& \int_{0}^{t} \int_{\Gamma_{C}}\left[F\left(u_{1 n}-g\right)-F\left(u_{2 n}-g\right)\right]\left[\left|u_{1 T}^{\prime}-\phi\right|-\left|u_{2 T}^{\prime}-\phi\right|\right] d \Gamma d s \\
& \quad \leqslant c \int_{0}^{t} \int_{\Gamma_{C}}\left|u_{1 n}-u_{2 n}\right|\left(1+\left|u_{1 n}-g\right|^{2}+\left|u_{2 n}-g\right|^{2}\right)\left|u_{1 T}^{\prime}-u_{2 T}^{\prime}\right| d \Gamma d s
\end{aligned}
$$




$$
\begin{aligned}
& \leqslant c \int_{0}^{t}\left\|u_{1}-u_{2}\right\|_{E}\left\|u_{1}^{\prime}-u_{2}^{\prime}\right\|_{E} d s \\
& \leqslant \frac{\alpha}{4} \int_{0}^{t}\left\|u_{1}^{\prime}-u_{2}^{\prime}\right\|_{E}^{2} d s+c \int_{0}^{t}\left\|u_{1}-u_{2}\right\|_{E}^{2} d s .
\end{aligned}
$$

Using the results of (6.3)-(6.7) in (6.2), we obtain

$$
\begin{aligned}
\mid u_{1}^{\prime}(t) & -\left.u_{2}^{\prime}(t)\right|_{H^{3}} ^{2}+\int_{0}^{t}|| u_{1}^{\prime}(s)-\left.u_{2}^{\prime}(s)\right|_{E} ^{2} d s \\
& \leqslant c \int_{0}^{t}\left|\xi_{1}-\xi_{2}\right|_{H}^{2} d s+c \int_{0}^{t}\left|u_{1}^{\prime}(s)-u_{2}^{\prime}(s)\right|_{H^{3}}^{2} d s+c \int_{0}^{t}\left\|u_{1}-u_{2}\right\|_{E}^{2} d s .
\end{aligned}
$$

An application of Gronwall's Inequality now yields

$$
\left|u_{1}^{\prime}(t)-u_{2}^{\prime}(t)\right|_{H^{3}}^{2}+\int_{0}^{t} \| u_{1}^{\prime}(s)-\left.u_{2}^{\prime}(s)\right|_{E} ^{2} d s \leqslant c \int_{0}^{t}\left|\xi_{1}-\xi_{2}\right|_{H}^{2} d s .
$$

Leaving this expression for the moment, we substitute $\xi_{1}$ and $\xi_{2}$ into (3.24) in turn, subtract the resulting equations and let this act on the difference $\xi_{1}-\xi_{2}$ to obtain

$$
\begin{gathered}
\frac{1}{2}\left|\xi_{1}(t)-\xi_{2}(t)\right|_{H}^{2}+\alpha \int_{0}^{t}|| \xi_{1}-\left.\xi_{2}\right|_{V} ^{2} d s-\beta \int_{0}^{t}\left|\xi_{1}-\xi_{2}\right|_{H}^{2} d s+\int_{0}^{t}\left\langle M_{1} u_{1}^{\prime}-M_{1} u_{2}^{\prime}, \xi_{1}-\xi_{2}\right\rangle d s \\
\leqslant-\int_{0}^{t}\left\langle S\left(u_{1}, u_{1}^{\prime}\right)-S\left(u_{2}, u_{2}^{\prime}\right), \xi_{1}-\xi_{2}\right\rangle d s .
\end{gathered}
$$

In arriving at (6.10) we have also used the Korn estimate (4.15). We now estimate the term involving $S$ as follows:

$$
\begin{aligned}
& \left|\left\langle S\left(u_{1}, u_{1}^{\prime}\right)-S\left(u_{2}, u_{2}^{\prime}\right), \xi_{1}-\xi_{2}\right\rangle\right| \\
& \quad \leqslant \int_{\Gamma_{C}}\left|F\left(x, u_{1 n}-g\right)-F\left(x, u_{2 n}-g\right)\right| s_{c}\left(\left|u_{1 T}^{\prime}-\phi\right|\right)\left|\xi_{1}-\xi_{2}\right| d \Gamma \\
& \quad+\int_{\Gamma_{C}} F\left(x, u_{2 n}-g\right)\left|s_{c}\left(\left|u_{1 T}^{\prime}-\phi\right|\right)-s_{c}\left(\left|u_{2 T}^{\prime}-\phi\right|\right)\right|\left|\xi_{1}-\xi_{2}\right| d \Gamma .
\end{aligned}
$$

The first integral on the right in (6.11) is estimated as in (6.5):

$$
\begin{aligned}
& \int_{\Gamma_{C}}\left|F\left(x, u_{1 n}-g\right)-F\left(x, u_{2 n}-g\right)\right| s_{c}\left(\left|u_{1 T}^{\prime}-\phi\right|\right)\left|\xi_{1}-\xi_{2}\right| d \Gamma \\
& \quad \leqslant c \int_{\Gamma_{C}}\left|u_{1 n}-u_{2 n}\right|\left(1+\left|u_{1 n}-g\right|+\left|u_{2 n}-g\right|\right)\left|\xi_{1}-\xi_{2}\right| d \Gamma \\
& \quad \leqslant c\left\|u_{1}-u_{2}||_{E}|| \xi_{1}-\xi_{2}\right\|_{V} \\
& \quad \leqslant c\left\|u_{1}-u_{2}\right\|_{E}^{2}+\frac{\alpha}{4}\left\|\xi_{1}-\xi_{2}\right\|_{V}^{2} .
\end{aligned}
$$

For the second integral on the right in (6.11) we note that since $F\left(\cdot, u_{2 n}-g\right) \leqslant c\left(1+\left|u_{2 n}-g\right|\right)$ and $u_{2} \in L^{\infty}(0, T ; E)$, it follows that $F\left(\cdot, u_{2 n}-g\right) \in L^{\infty}\left(0, T ; L^{4}\left(\Gamma_{C}\right)\right)$. Using this fact, (6.1) and the multi-Hölder inequality, we obtain

$$
\begin{gathered}
\int_{\Gamma_{C}} F\left(x, u_{2 n}-g\right)\left|s_{c}\left(\left|u_{1 T}^{\prime}-\phi\right|\right)-s_{c}\left(\left|u_{2 T}^{\prime}-\phi\right|\right)\right|\left|\xi_{1}-\xi_{2}\right| d \Gamma \\
\leqslant c\left(\int_{\Gamma_{C}}\left|u_{1 T}^{\prime}-u_{2 T}^{\prime}\right|^{2} d \Gamma\right)^{1 / 2}\left(\int_{\Gamma_{C}}\left|\xi_{1}-\xi_{2}\right|^{4} d \Gamma\right)^{1 / 4}
\end{gathered}
$$




$$
\begin{aligned}
& \leqslant c\left\|u_{1}^{\prime}-u_{2}^{\prime}\right\|_{E}\left\|\xi_{1}-\xi_{2}\right\|_{V} \\
& \leqslant \frac{\alpha}{4}\left\|\xi_{1}-\xi_{2}\right\|_{V}^{2}+c\left\|u_{1}^{\prime}-u_{2}^{\prime}\right\|_{E}^{2}
\end{aligned}
$$

Using the results of (6.11)-(6.13) in (6.10) and rearranging terms yields

$$
\begin{aligned}
& \frac{1}{2}\left|\xi_{1}(t)-\xi_{2}(t)\right|_{H}^{2}+\frac{\alpha}{2} \int_{0}^{t}\left\|\xi_{1}-\xi_{2}\right\|_{V}^{2} d s \\
& \quad \leqslant \beta \int_{0}^{t}\left|\xi_{1}-\xi_{2}\right|_{H}^{2} d s+c \int_{0}^{t}\left\|u_{1}-u_{2}\right\|_{E}^{2} d s+c \int_{0}^{t}\left\|u_{1}^{\prime}-u_{2}^{\prime}\right\|_{E}^{2} d s .
\end{aligned}
$$

Using (6.9) in this yields

$$
\frac{1}{2}\left|\xi_{1}(t)-\xi_{2}(t)\right|_{H}^{2}+\frac{\alpha}{2} \int_{0}^{t}|| \xi_{1}-\left.\xi_{2}\right|_{V} ^{2} d s \leqslant c \int_{0}^{t}\left|\xi_{1}-\xi_{2}\right|_{H}^{2} d s .
$$

Now we apply Gronwall's inequality to conclude that $\xi_{1}=\xi_{2}$. Then (6.9) gives $u_{1}^{\prime}=u_{2}^{\prime}$ which implies $u_{1}=u_{2}$. This proves Theorem 6.1 .

\section{Conclusions}

We have established the existence, and under additional conditions, uniqueness of a weak solution to a problem that involves frictional contact accompanied by heat generation. This opens the way to further investigate properties of this model from both theoretical and applied points of view. We remark that the methods used in this paper may be used to treat other types of boundary conditions than those presented in (2.5)-(2.7). For example, instead of the fixed temperature condition (2.7), we may have a heat exchange condition on all or part of $\Gamma_{D} \cup \Gamma_{N}$. We could also allow the temperature functions $\theta_{b}$ and $\theta_{R}$ to be time dependent.

We can also establish existence under different growth assumptions on $F$ and $s_{c}$; mathematically, all that is required is the existence of nonnegative numbers $a$ and $b$ such that $a+b=1$ and $F(x, r) \leqslant c\left(1+|r|^{a}\right)$ and $s_{c}(x, r) \leqslant c\left(1+|r|^{b}\right)$. The fact that restrictions are imposed on the product of $F$ and $s_{c}$ rather than on each factor individually may possibly be related to the fact that within the framework of linear thermoelasticity frictional heat generation leads to an increase in the contact pressure and vice versa. In any case, it is likely that the model breaks down in the presence of large slip velocities and so, practically speaking, these growth assumptions are not as restrictive as would first appear.

One of the issues that requires further investigation in this area is the determination of conditions under which the process which induces growth in the contact pressure and heat generation becomes unstable. Such a situation may arise, for example, in braking systems, where the growth of temperature may lead to a softening of the system material, leading to subsequent loss of braking power. A related issue is the development of hot spots on the contacting surface. It is possible that an initial inhomogeneity on the contact surface may lead to a situation where only a small portion of the body comes into contact with the foundation and is thus subjected to frictional heating, thermoelastic expansion and growth of contact pressure. In such a manner a hot spot is created. The results of this paper give only a preliminary insight into these difficult questions. For example, we can deduce from Lemma 4.5 and standard regularity theorems that the displacement $u$ is 
in $C\left(0, T ;\left(H^{1}(\Omega)\right)^{3}\right)$ and the temperature $\theta$ is in $C\left(0, T ;\left(L^{2}(\Omega)\right)^{3}\right)$ but these results do not contain sufficient spatial regularity to rule out the existence of hot spots on the contacting surface.

We had earlier remarked that the model presented here allows for coefficients of friction which may vary with the contact stress. In fact, our methods may also be used to analyze a situation where the coefficient of friction depends on the slip between the body and the foundation. Friction laws of this type are employed in modelling earthquakes [23-25].

Finally, we point out that the approximate problem described in $\S 4$ may serve as the basis for a finite element numerical algorithm, which may be used to generate numerical simulations of the original problem. One advantage of working with such an algorithm is that its convergence properties have been established. By contrast, some algorithms used in frictional contact problems as the basis for computer codes do not have established convergence properties and consequently must frequently be manipulated so as to achieve a seemingly satisfactory solution.

\section{References}

[1] Germain, P. (1973) Mecanique de Milieux Continus, Masson, Paris.

[2] Duvaut, G. \& Lions, J. L. (1976) Inequalities in Mechanics and Physics, Springer-Verlag, Berlin.

[3] Maugin, G. A. (1992) The Thermomechanics of Plasticity and Fracture, Cambridge University Press.

[4] Do, C. (1980) On the dynamic deformation of a bar against an obstacle, in Variational Methods in the Mechanics of Solids, S. Nemat-Nasser (ed.), Pergamon, Oxford, 237-241.

[5] Кıм, J. U. (1990) A one-dimensional dynamic contact problem in linear viscoelasticity. Math. Methods Appl. Sci. 13, 55-79.

[6] Kuttler, K. L. (1994) A dynamic contact problem in viscoelasticity. Adv. Math. Sci. Appl. 4, $297-312$.

[7] Andrews, K. T., Shi, P., Shillor, M. \& Wright. S. (1993) Thermoelastic contact with Barber's heat exchange condition. Appl. Math. Optim. 28, 11-48.

[8] ANDRews, K. T., Shillor, M. \& WRight, S. (1994) A hyperbolic-parabolic system modeling the thermoelastic impact of two rods. Math. Meth. Appl. Sci. 17, 901-918.

[9] ElLiott, C. M. \& QI, T. (1994) A dynamic contact problem in thermoelasticity. Nonlinear Analysis TMA 23, 883-898.

[10] KuttLeR, K. L. \& ShiLlor, M. (1995) A one-dimensional thermoviscoelastic contact problem. Nonlinear World 2, 355-385.

[11] Oden, J. T. \& Martins, J. A. C. (1985) Models and computational methods for dynamic friction phenomena. Comput. Meth. Appl. Mech. Engrg. 52, 527-634.

[12] Back, N., Burdekin, M. \& Crowley, A. (1973) Review of the research on fixed and sliding joints. Proceedings 13th International Machine Tool Design and Research Conference, S. A. Tobias \& F. Koenigsberger (eds.), MacMillan, London, 87-97.

[13] Klarbring, A., Mikelic, A. \& Shillor, M. (1988) Frictional contact problems with normal compliance. Int. J. Eng. Sci. 26, 811-832.

[14] KutTLER, K. L. (1997) Dynamic friction contact problems for general normal and friction laws. Nonlin. Anal. TMA 28, 559-575. 
[15] Martins, J. A. C. \& Oden, J. T. (1987) Existence and uniqueness results for dynamic contact problems with nonlinear normal and friction interface laws. Nonlinear Analysis TMA 11, 407-428.

[16] Figueiredo, I. \& Trabucho, L. (1995) A class of contact and friction dynamic problems in thermoelasticity and in thermoviscoelasticity. Int. J. Eng. Sci. 33, 45-66.

[17] Kikuchi, N.\& Oden, J. T. (1988) Contact Problems in Elasticity, SIAM, Philadelphia.

[18] Kuttler, K. L. (1986) Time dependent implicit evolution equations. Nonlin. Anal. TMA 10, $447-463$

[19] Kuttler, K. L. (1990) Initial-boundary value problem for the displacement in an isothermal viscous gas. Nonlin. Anal. TMA 15, 601-623.

[20] Aubin, J. B. (1972) Approximation of Elliptic Boundary Value Problems. Wiley.

[21] Lions, J. L. (1969) Quelques Methodes de Resolution des Problemes aux Limites Non Lineaires, Dunod, Paris.

[22] Oleinik, O. A., Shamaev, A. S. \& Yosifian, G. A. (1992) Mathematical Problems in Elasticity and Homogenization, North-Holland, New York.

[23] Ionescu, I. R. \& Paumier, J. C. (1996) On the contact problem with slip displacement dependent friction in elastostatics. Int. J. Eng. Sci. 34, 471-491.

[24] Ionescu, I. R. \& Paumier, J. C. (1994) On the contact problem with slip rate dependent friction in elastodynamics. Eur. J. Mech, A/Solids 13, 555-568.

[25] Scholz, C. H. (1990) The Mechanics of Earthquakes and Faulting. New York. Cambridge University Press. 